\title{
AVOIDANCE OF EARTH OBSTACLES FOR INTELLIGENT EXCAVATOR
}

\author{
Jangho Bae, Chang Seup Lee, and Daehie Hong* \\ Department of Mechnical Engineering, Korea University, Seoul, Korea \\ *Corresponding author(dhhong@korea.ac.kr)
}

\begin{abstract}
In this paper, we develop an algorithm for avoidance of earth obstacles using model-reference impedance control for autonomous excavator. There have been many research in the past regarding autonomous excavation, which were only concerned about position control, force control and path generation. However in order to apply the control system or algorithm to the actual autonomous excavation, it is essential that prediction and avoidance of potential obstacle must be carried out. When earth obstacles such as rocks and tree roots are detected by force/torque sensor, accidents can be easily prevented by halting the excavation task using a simple fail-safe algorithm. However, it would be more desirable to control the unpredictable bucket/earth obstacle interactions intelligently on-line while continuing the excavation task instead of stopping. Impedance control is a control scheme, which is used for defining dynamic relationship between end-effector and environment when the end-effector is constrained in the environment. In this case end-effector would be the bucket tip and environment would be the earth obstacle. In this paper a novel algorithm was developed which realize the obstacle avoidance of autonomous excavator that can regulate bucket force in real time.

For the experimental use, hydraulic manipulator which resembles commercial excavator was designed. Two kinds of sensor, potentiometers and 6 axis force/torque sensor, were used to provide position and bucket tip force feedback information. Experimental results indicate that bucket has avoided earth obstacle smoothly without large force and all desired task is completed.
\end{abstract}

Keywords: Autonomous Excavator, Impedance Control, Avoidance of Earth Obstacles

\section{INTRODUCTION}

Excavation is of importance in digging, trenching, leveling and general earthworks. However, human operators require ten to fifteen years of experience before they can be considered experts. Also their works are often dirty, strenuous and repetitive. In addition, excavation environment is recognized as one of the most hazardous construction environment. Therefore, automation of excavation is essential to resolve the potential risks inherited in excavation environment and to improve work efficacy and time efficiency. So, many efforts have been made on development of automated excavator [1], [2], [3]. The previous studies on autonomous excavator were focused on improvement of position control performance by using analysis of the kinematics and dynamics, force tracking with impedance control, interaction between the bucket and soil and optimal path planning for excavation [4], [5], [6], [7], [8]. However in order to achieve a high level autonomous excavation, it is essential that buried obstacles is predicted and avoided or removed. When earth obstacles such as rocks are detected by force/torque sensor, accidents can be easily prevented by halting the excavation task using a simple fail-safe algorithm. However, it would be more desirable to control the unpredictable interaction between bucket and obstacle intelligently real time while continuing the excavation task instead of stopping. In university of Arizona, studies related avoidance obstacles have been conducted [9]. This studies presented implements of excavation tasks as sequences of behaviors and behaviors as sequences of actions. Especially, the 
excavation actions of bucket/obstacles interaction are specified using fuzzy logic rules acquired from human experience and heuristics.

In this paper, a novel algorithm for avoidance of earth obstacles using model-reference impedance control is developed for autonomous excavator. When excavator is digging the soil, the impedance, which is stiffness and damping value, is adjusted to track desired path. If interaction force between bucket and obstacles reaches a threshold force because bucket contacts unexpected obstacle, by adjusting the impedance, the interaction force is maintained less than threshold force and then bucket can avoid an obstacle using the earth obstacles avoidance algorithm which is developed in this paper. This paper is organized as follows. In chapter 2, a model-reference impedance control which is suitable for hydraulic system is referred. Chapter 3 deals with behavior of bucket while bucket contacting an obstacle and addresses the algorithm for earth obstacles avoidance. Chapter 4 describes the designed hydraulic manipulator which resembles a commercial excavator for experimental use. Chapter 5 presents experimental results about the avoidance of earth obstacles algorithm. Finally, in chapter 6, conclusions are addressed.

\section{MODEL-REFERENCE IMPEDANCE CONTROL}

The term 'impedance control' was first introduced by Neville Hogan [10], and is a general approach to the control of manipulation. Impedance control is intended to encompass the traditional positioning tasks performed by manipulators while including the capability to control static and dynamic interactions between the manipulator and the environment. The role of impedance control is, therefore, to maintain a desired dynamic relationship, referred to as the target impedance, between the end-effector position and the end-effector/environment contact force as shown in Fig. 1. The most common implementation of impedance control computes a control torque which transforms the existing robot dynamics into a desired form. In robotics literature, this computed torque technique is referred to as 'torque-based impedance control'. In this torque-based

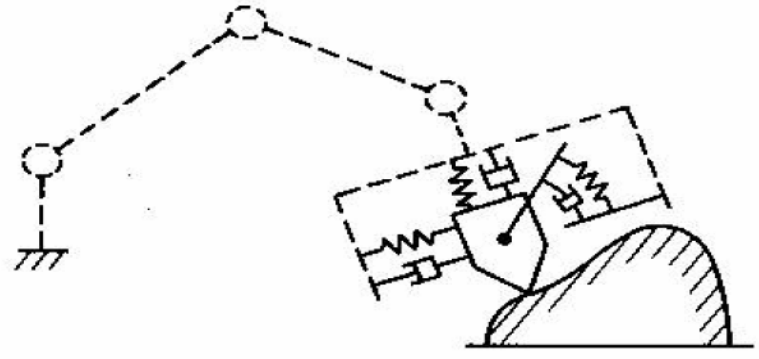

Fig. 1 Desired dynamic relationship on impedance control

impedance control, it is seen that the technique highly depends on dynamic characteristics of the manipulator. However, most industrial manipulators are difficult to obtain the dynamic characteristics which consist of inertia, centrifugal/Coriolis forces, gravitational forces and friction forces. Manipulator with hydraulic actuators also cannot apply the control torque to each joint of manipulator. An alternative implementation of the torque-based impedance control is a model-reference impedance control which is shown in Fig. 2 [6]. In this implementation, the interaction force sensed by force sensor and the commanded input position are used to compute the corresponding motion of a second order impedance model. The existing position controller of manipulator is used to track the model's output motion. The model-reference impedance controller requires neither a dynamic model of robot or torque controlled actuators. This type of impedance control is suitable for hydraulic manipulator such as excavator robot.

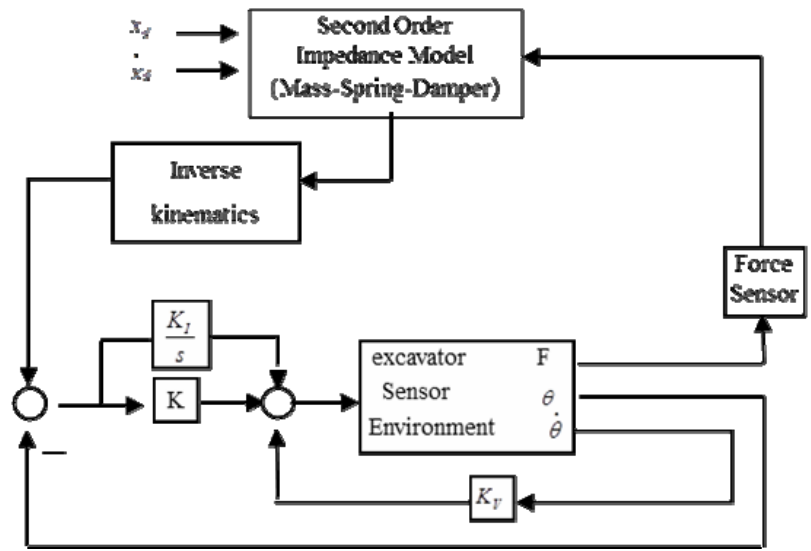

Fig. 2 Model-reference impedance control 


\section{ALGORITHM FOR AVOIDANCE OF EARTH OBSTACLES}

Human operator detects unpredictable obstacles by sense of handling levers, movement of excavator arms and sounds of pumps and so on. In intelligent excavation system, obstacles can be detected by sensing a sudden increase in interaction force using force/torque sensor. A general path of excavation is classified into three consecutive paths, penetration, drag and curl, as shown in Fig. 3. While the bucket is digging that path, constraint condition has to be set up, namely, it means that an attack angle of bucket tip is larger than a processing one, so it could avoid a situation as shown in Fig. 4(b). On the excavation task, when the bucket tip encounters the obstacle, the environment could be considered an infinite stiffness environment unlike soil. It results in failure of the excavator robot. The impedance control for that environment helps control the forces which were affected the excavator robot, so it prevents mechanical defect. Fig. 5 shows a behavior of the bucket when the bucket encounters an obstacle with impedance controller. The bucket tip can't follow the desired position due to the obstacles, and stayed the contact point as Fig. 5. In addition,

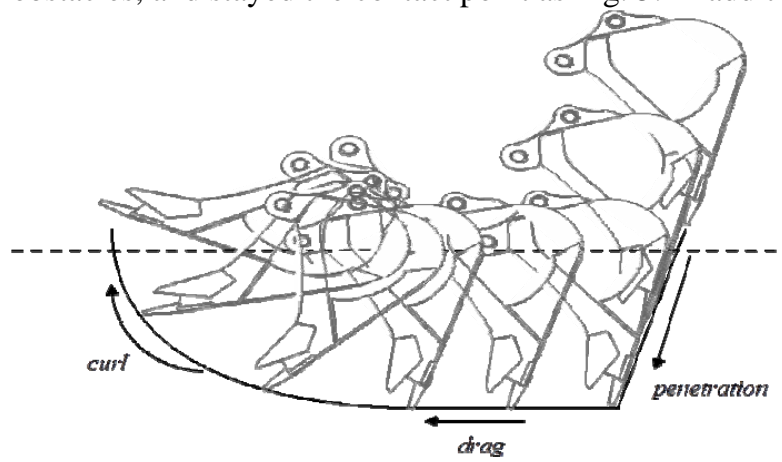

Fig. 3 Typical path of excavation

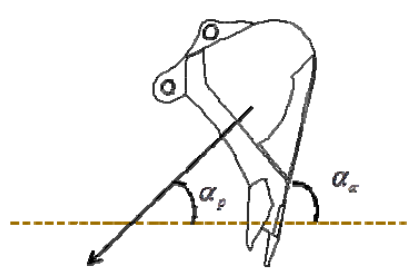

(a)

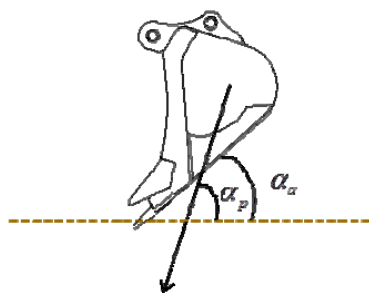

(b)
Fig. 4 2D motion behavior of bucket when contacting obstacles

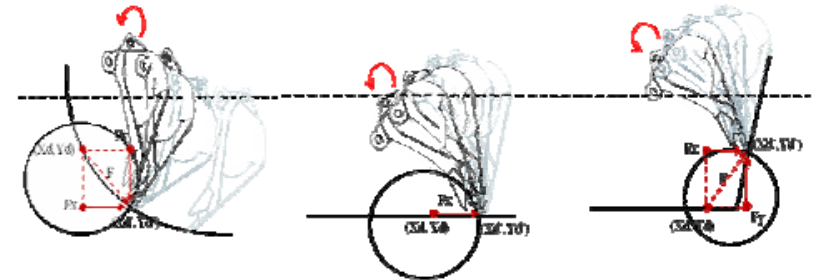

(a)

(b)

(c)

Fig. $52 \mathrm{D}$ motions of bucket when bucket is contacting obstacles with impedance control in three paths

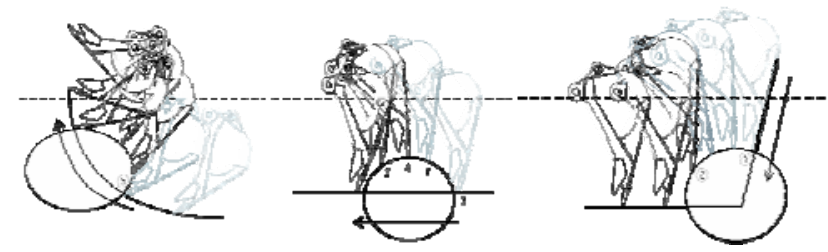

(a)

(b)

(c)

Fig. 6 2D motions of bucket which handle the obstacle in three paths using the algorithm for earth obstacles

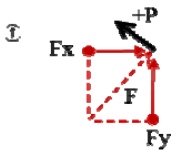

3.<smiles>[10BH][10BH]</smiles>

?

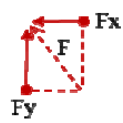

s.

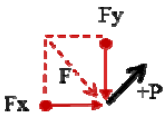

Fig. 7 Contacting force at (1) - (5) of Fig. 6

the angle of the bucket will be rotated, in order to avoid constraint conditions which mentioned before. At that time, there is no large force which acts on the bucket tip by adjusting the appropriate impedance. However, this behavior doesn't control the obstacle effectively, even though it prevents large force from acting on the excavator. Using this property, the algorithm for earth obstacles avoidance can be developed. Fig 6 shows the desired motion of handling the obstacles compliantly. In Fig 6, the point (1), (3) and (5) are the part which cannot proceed because of the obstacles. And the interaction force between bucket and obstacle of each point is shown in Fig 7. Here, command the bucket to process forward to $+\mathrm{P}$ direction which is a right normal vector of a resultant force F. It will move to forward $\mathrm{P}$ to avoid stopping phenomenon. At the 
point (2) and (4), the bucket can through the obstacles compliantly only with the proper impedance adjustment. In conclusion, the novel algorithm has come from the integration of both added moving command toward to the right normal direction of the force where the bucket is acted, and the impedance control.

\section{A DESIGNED HYDRAULIC MANIPULATOR}

Controlling real excavator is not easy. Because the entire pilot operated, semi-automated valve system must be replaced with electronic valve module. More importantly, application of any premature algorithm directly to real excavator seems too dangerous in term of safety and monetary issues. In this paper, hydraulic manipulator which resembles commercial excavator was, therefore, designed for experimental use as shown in Fig. 8. Each cylinder displacement, lengths of each link and bucket

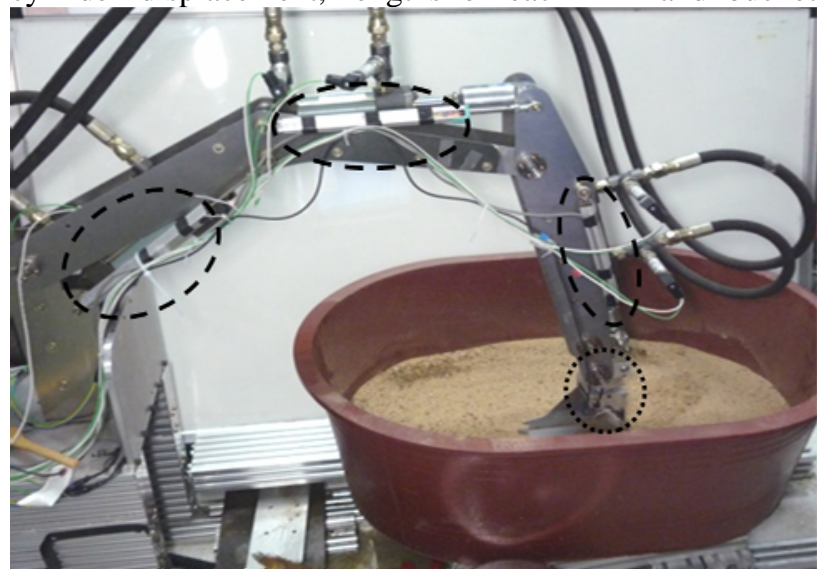

Fig. 8 Custom-designed hydraulic manipulator

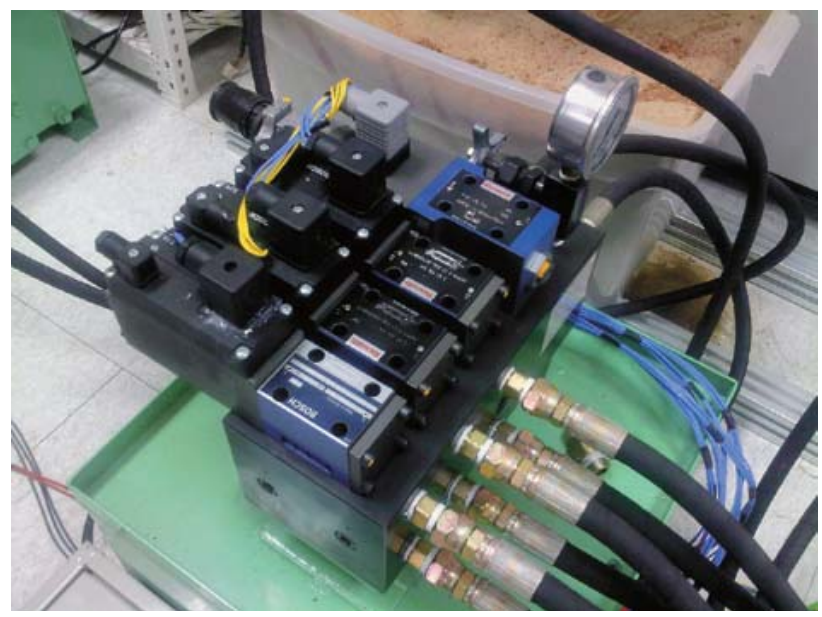

Fig. 9 Valve module volume was scaled by approximately 1:10. Each cylinder was actuated by three servo solenoid valves which were used to control flow rate of hydraulic oil electrically. Fig. 9 shows the servo solenoid valves that were equipped with high precision linear variable differential transformer (LVDT) with response time less than 10ms. Because of this fast response time, dead band effect can be negligible. Two kinds of sensor were used to provide feedback information. Position feedback information was obtained using potentiometers. Same as the number of cylinders, three cylinder-rod type high precision potentiometers which have independent linearity of $0.05 \%$ and infinite resolution were installed parallel with each cylinder assembly. A 6 axis force/torque sensor was attached between end-effector and bucket assembly to provide bucket tip force information.

\section{EXPERIMENT RESULT}

In this chapter, various experiments were conducted to demonstrate that the proposed algorithm of earth obstacle avoidance. Based on a general excavation path, by using the obstacle avoidance algorithm, three tests for avoiding a cylindroid obstacle was carried out separately in three paths, penetrate, drag and curl. On Fig. 10, 12 and 13, a dotted line, line and bold dotted line mean desired position, actual position and cylindroid obstacle, respectively. Here, the bucket moves from left to right. First, Fig. 10 shows a motion of bucket, when bucket encounters the obstacle for penetrate path. When the Bucket first contacts the obstacles, usually the interaction force is produced forward to Fig. 7 (1)-direction, so the right normal direction moving command is added to bucket tip, because of the obstacles avoiding algorithms. Now it is possible to see the compliant motion following the surface of obstacles without stopping at any point. Fig. 11 shows the then interaction forces of $\mathrm{x}$ and $\mathrm{y}$ direction, separately. The interaction force is not over threshold $50 \mathrm{~N}$, without sudden change, even while the bucket is handling the obstacles perfectly. Next case is that the bucket encounters the obstacles in the drag path. The force is generated forward to the direction which shows in Fig. 7(3). According to the avoid obstacle algorithm, the moving command was 


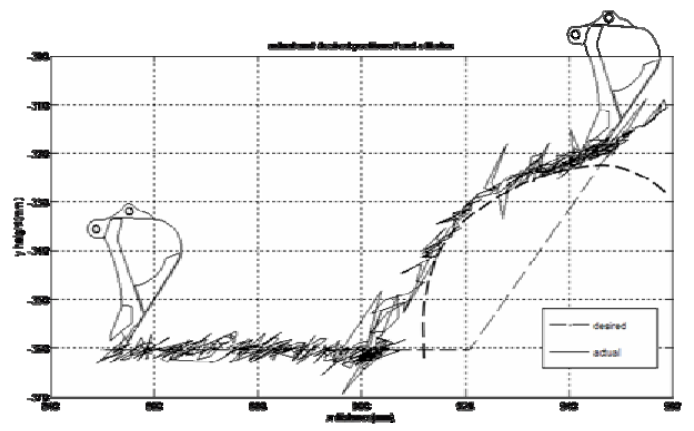

Fig. 10 Bucket motion for penetration

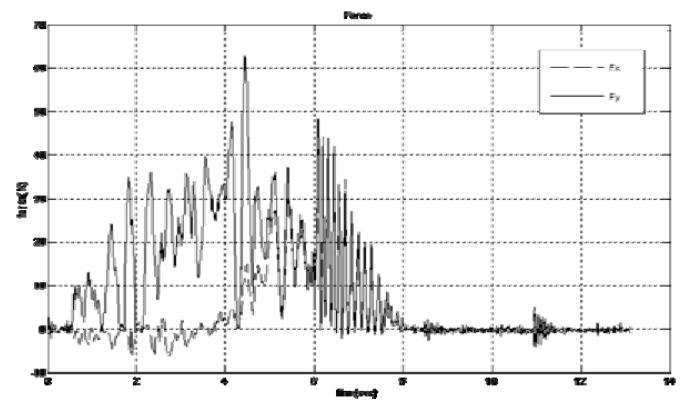

Fig. 11 Contact force for penetration

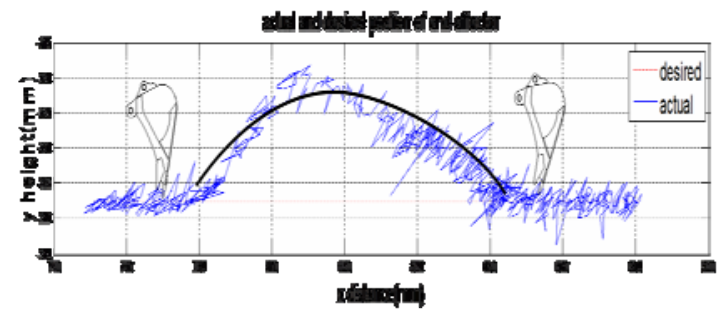

Fig. 12 Bucket motion for drag

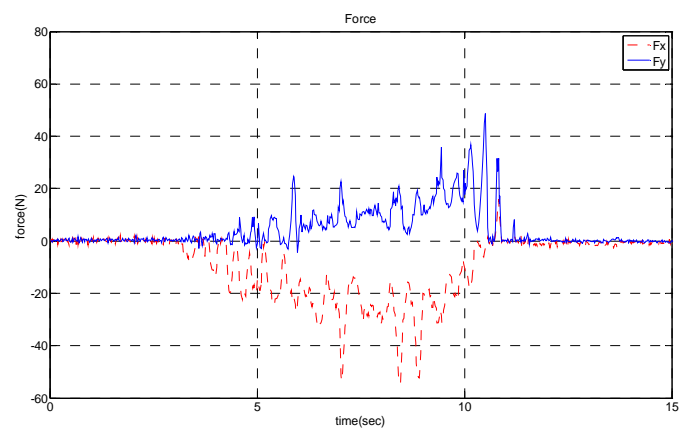

Fig. 13 Contact force for drag

added toward y-direction, and the bucket was following the surface of the obstacle. After x-coordinate of bucket is over $840(\mathrm{~mm})$ in Fig. 12, although the bucket tries to back to the desired position, it cannot because of the resist of obstacle. At that time the direction of forces is same as the Fig. 7(1).

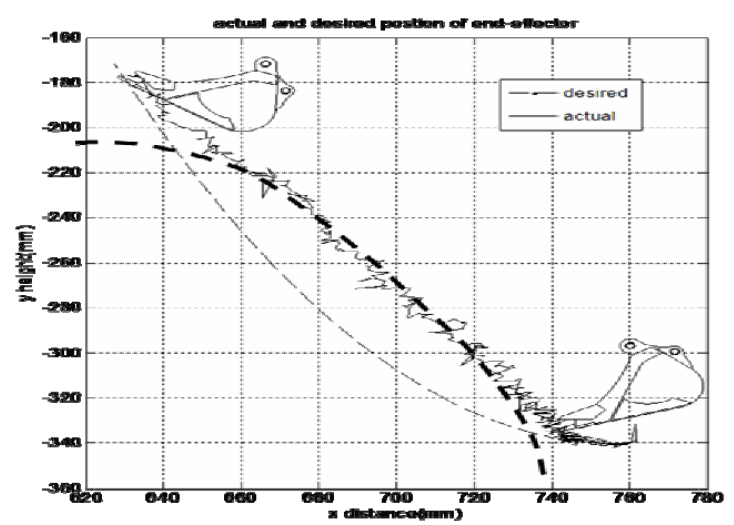

Fig. 14 Bucket motion for curl

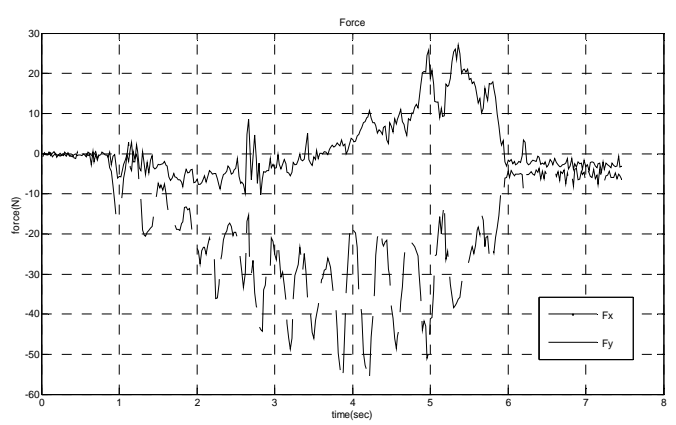

Fig. 15 Contact force for curl

The moving command which is by algorithm is added as first case, and the bucket follows the surface of the obstacle. The $\mathrm{x}$ and $\mathrm{y}$ direction force are in Fig 13. At last, in case of encounter the obstacle at curl path, the motion of bucket is similar to the former 2 cases. The Bucket processes follow the obstacle as shown in Fig. 14. Mostly the bucket is acted by forces which are either Fig. 7 (3) or (5), and the extra moving command is added as well by the algorithm of avoidance obstacles. As same as former cases, the force is not over certain threshold as shown in Fig. 15.

The experiment results show that the obstacle is able to be controlled by using the algorithm of avoidance obstacles regardless of any paths. Also by adjusting impedance, we can set the desired threshold force.

\section{CONCLUSION}

In this paper, we developed an algorithm of earth obstacles avoidance using the impedance controller. First of all, we applied model-reference impedance control, which is 
suitable for manipulator with hydraulic actuators, to hydraulic manipulator designed in Chapter 4. And then, experiments for earth obstacles avoidance was conducted under the three excavation paths. As verified by experiment, when the bucket contacts the obstacle, it can avoid obstacle smoothly less than a threshold force. While the excavation robot is working in excavation task, it secures the safety of excavator, and completes the excavation task even though bucket contacts the obstacles without stopping the work or going back to the first step. Therefore the overall efficiency of the autonomous excavation system will be improved. Furthermore this research will be the basic of studies of the obstacle removal.

\section{ACKNOWLEDGEMENT}

This research was supported by a grant (code:06 high technology fusion 001) from the Unified and Advanced Construction Technology Program that was funded by the Ministry of Land, Transport and Maritime Affairs of Korean government and Brain Korea 21.

\section{REFERENCES}

[1] Singh, S., "Synthesis of Tactical Plans For Robotic Excavation”, Doctoral Dissertation Tech. Report CMU-RITR-95-27, Robotics Institute, Carnegie Mellon University, January, 1995.

[2] Joseph, G. F., "Development of a Haptic Backhoe Testbed”, The Degree of Master of Science in Mechanical Engineering, Georgia Tech. Thesis and Dissertations, May 2004.

[3] Stentz, A., Bares, J., Singh, S. and Rowe, P., “A Robotic Excavator for Autonomous Truck Loading”, Autonomous Robots, Vol. 7(3), pp. 175-186, 2003.

[4] A.J., Koivo, M., Thoma, E., Kocaoglan, J. and Andrade-Cetto, "Modeling and control of excavator dynamics during digging operation”, J. Aerosp. Eng. Vol. 9, pp. 10-18, 1996.
[5] Quang, N. H., "Robust Low Level Control of Robotic Excavation”, Australian Centre for Field Robotics, A Thesis of Doctor of Philosophy, The University of Sydney. [6] Ha, Q.P., Nquyen, Q.H., Rye, D.C. and Durrant-Whyte, "Impedance control of a hydraulically actuated robotic excavator”, Automation in Construction, Vol. 9, pp. 421435, 2000

[7] Luengo, O., Singh, S. and Cannon, H., "Modeling and Identification of Soil-tool Interaction in Automated Excavation”, International Conf. on Intelligent Robotic System, 1998.

[8] Park, H. J., “A task-planning for Autonomous Excavation under Robot Underground Environment Interactions”, M. S. Graduate paper, Department of Mechanical Engineering, University of Korea, 2009.

[9] Shi, X., Wang, F. and Lever, P. J. A., "Experience Results of Robotic Excavation Using Fuzzy Behavior Control”, Control Eng. Practice,, Vol. 4(2), pp. 145-152, 1996.

[10] Hogan, N., "Impedance Control: An Approach to manipulation, part I”, ASME Journal of Dynamics Systems, Measurement, and Control, Vol. 107, pp. 1-24, 1985.

[11] Field, G. and Stepanenko, Y., "Model Reference Impedance Control of Robotic Manipualtors”, Proc.IEEE Pacific Rim Conference, Vol. 2, pp. 614-617, 1993. 